\title{
DISEÑO DE UN INDICADOR ADELANTADO PARA LA ACTIVIDAD ECONÓMICA DE COSTA RICA ${ }^{1}$
}

\author{
DESIGN OF A LEADING INDICATOR FOR COSTA RICAN ECONOMIC ACTIVITY
}

\section{Carlos Chaverri Morales ${ }^{2}$ \\ Diana Van Patten Rivera ${ }^{3}$}

\begin{abstract}
Resumen
Este documento presenta los resultados de la estimación de tres indicadores adelantados de los puntos de giro de la actividad económica en Costa Rica. Lo anterior sigue la metodología propuesta por la Organización para la Cooperación y el Desarrollo Económico (OCDE). Se seleccionó el Índice Mensual de Actividad Económica (IMAE) como variable de referencia y se analizaron 270 variables que contienen información de los sectores monetario, real, mercado laboral, así como índices de precios, indicadores del sector externo y variables del sector fiscal. La información del sector real se desagregó en tres niveles que incluían una clasificación con datos a nivel de la Clasificación Industrial Internacional Uniforme (CIIU) con dos dígitos, información del sector agrícola de acuerdo con la Clasificación Central de Productos (CCP) e información a nivel de producto del sector manufacturero. Para cada nivel de agregación se desarrolló un indicador adelantado, con adelantos promedio de entre 7 y 12 meses respecto a la variable de referencia.
\end{abstract}

Palabras claves: Modelos de series temporales; fluctuaciones y ciclos económicos.

Doi: http://dx.doi.org/10.15359/eys.19-46.4

Recibido el 11 de setiembre del 2014. Aceptado el 28 de noviembre del 2014. Publicado el 23 de diciembre del 2014.

${ }^{1}$ Las ideas expresadas en este documento son de los autores y no necesariamente representan las del Banco Central de Costa Rica (BCCR).

${ }^{2}$ Funcionario, BCCR, chaverrimc@bccr.fi.cr

${ }^{3}$ Funcionaria, BCCR, vanrd@bccr.fi.cr

56

Carlos Chaverri Morales y Diana Van Patten Rivera

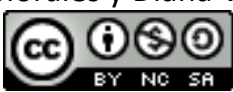

Artículo protegido por licencia Creative Commons 


\begin{abstract}
This paper presents the results of estimating three leading indicators for the turning points of the economic activity in Costa Rica. This was done following the methodology proposed by the Organization for Economic Cooperation and Development (OECD). The Monthly Economic Activity Index (IMAE in Spanish) was selected as the reference variable. A total of 270 data series were analyzed including monetary, real and job market variables, as well as price indices, external sector indicators and fiscal sector variables. The real sector information was disaggregated into three levels, which included the classification of data at an industrial level using the International Standard Industrial Classification (ISIC) with two digits, information from the agricultural sector based on the Central Product Classification (CPC) and information from the manufacturing sector. A leading indicator was developed for each level of aggregation, resulting in average leads of 7 to 12 months compared to the reference variable.
\end{abstract}

Keywords: Time-Series Models; Business Cycles and Economic Fluctuations.

\title{
Introducción ${ }^{4}$
}

Los estudios recientes sobre la actividad económica costarricense se han concentrado en dos temas particulares: i) la identificación de la cronología de los puntos de giro del ciclo económico (Chaverri, 2011) y ii) la documentación de las principales regularidades empíricas de la economía (Chaverri y Rodriguez, 2013).

Sin embargo, las necesidades de ampliar las herramientas analíticas para estudiar la coyuntura económica ha propiciado el diseño de instrumentos que permiten elaborar escenarios de proyección de las principales variables macroeconómicas conjuntamente con el desarrollo y evaluación de indicadores para la identificación temprana de presiones inflacionarias mediante técnicas de nowcasting.

Para lo primero se ha desarrollado un modelo satélite para generar proyecciones de los componentes del producto interno bruto según el enfoque del gasto, herramienta que

\footnotetext{
${ }^{4}$ Cifras preliminares a octubre 2013. Los autores agradecen las observaciones y recomendaciones proporcionadas por el asesor doctor Armando Sánchez Vargas; investigador y profesor de la Universidad Autónoma Nacional de México y del CAPTAC-DR/FMI.
}

Carlos Chaverri Morales y Diana Van Patten Rivera

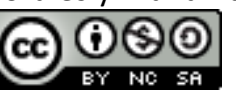

Artículo protegido por licencia Creative Commons 
complementa los resultados generados por el Modelo Macroeconómico de Proyección Trimestral (MMPT) y las estimaciones del Departamento de Estadística Macroeconómica. Para lo segundo y como lo es la práctica en muchos bancos centrales e institutos de estadística se han dedicado esfuerzos para desarrollar indicadores cíclicos que permitan comprender la dinámica y la capacidad predictiva del ciclo económico ${ }^{5}$.

Estos indicadores tienen como objetivo determinar si series temporales muy heterogéneas entre sí pueden proporcionar de forma conjunta o individual información relacionada con el ciclo económico. Es por lo anterior que a partir del trabajo pionero de Arthur Burns y Wesley Mitchell ${ }^{6}$ diversas instituciones han construido desde finales de la década de los 30 indicadores coincidentes, rezagados y adelantados.

Los indicadores coincidentes son aquellos que presentan un comportamiento sincrónico al ciclo económico de referencia, el cual confirma la evolución contemporánea de la actividad económica. Un indicador rezagado tiene como característica que responde con cierto desfase a los movimientos cíclicos de la economía, con lo cual tienen un papel confirmativo al comprobar las oscilaciones del ciclo en meses posteriores.

Por su parte, un indicador adelantado tiene como objetivo brindar información de manera anticipada acerca del comportamiento de un fenómeno económico particular. Estos pueden ser representados por una única serie, la cual a su vez debe de guardar una alta correlación con respecto a un fenómeno económico (usualmente la producción o la inflación).

Contrario a la práctica de utilizar una única variable como insumo de análisis, otra corriente metodológica sugiere la construcción de indicadores cíclicos compuestos, estos se construyen a partir de la síntesis de la información cíclica contenida en un conjunto de variables que están altamente correlacionadas con el fenómeno que se intenta estudiar. En este grupo destacan los indicadores desarrollados y publicados por entidades como la Reserva Federal de Chicago, la Organización para la Cooperación y el Desarrollo Económicos (OCDE), el Instituto de Estadísticas y Geografía de México (INEGI), entre otros.

La información que proveen los indicadores cíclicos debe de analizarse en términos de su capacidad predictiva o confirmativa de los fenómenos económicos y no como una herramienta para medir la intensidad o magnitud de los cambios coyunturales que experimenta la economía.

Este documento presenta los resultados de tres indicadores mensuales adelantados para la economía costarricense, en donde se utiliza como variable de referencia el Índice Mensual de

\footnotetext{
${ }^{5}$ Para mayor detalle ver Gallardo y Pendersen (2007) y Zarnowitz (1992)

${ }^{6}$ Particularmente se hace referencia a lo mencionado por Stock y Watson (1989), quienes explican que durante el año 1937 estos autores desarrollaron el primer grupo de indicadores cíclicos para la economía de Estados Unidos. 58
}

Carlos Chaverri Morales y Diana Van Patten Rivera

Artículo protegido por licencia Creative Commons 
Actividad Económica (en adelante, IMAE) bajo un enfoque cualitativo ${ }^{7}$. La selección de dicha variable y la periodicidad del indicador propuesto se justifica en las recomendaciones que hace el Instituto Nacional de Estadísticas de España y que son recopiladas por Abad, Cristóbal y Quilis (1994) en cuanto a que dicha variable satisface los siguientes requisitos: i) representa adecuadamente la actividad económica agregada, ii) posee un significado económico claro, iii) es elaborado de forma fiable y posee una señal cíclica identificable, iv) está disponible con prontitud y v) se construye con periodicidad mensual.

Los indicadores construidos utilizan la información procedente de un gran número de indicadores individuales, con distintos niveles de agregación que forman parte de diversos sectores de la economía. La metodología que se usa para la construcción de los indicadores compuestos sigue las recomendaciones propuestas por la $\underline{\operatorname{OCDE}(1998,2005)^{8}}$, las cuales, además, se complementan con una serie de sugerencias metodológicas emitidas por otras instituciones para garantizar el uso de series de tiempo que se apeguen a las mejores prácticas estadísticas.

Luego de esta introducción, en la segunda sección se presenta una breve reseña con algunos estudios similares para otras economías. En la tercera sección se explicará la metodología empleada y los criterios para seleccionar las variables que componen la base de datos y el indicador cíclico. En la cuarta y quinta sección se explica la metodología que se sigue para la selección de variables y la construcción del indicador propuesto. Las secciones sexta y sétima describen los datos y se mencionan los principales resultados. Finalmente, la octava sección contiene las principales conclusiones de la investigación y las recomendaciones del caso.

\section{Antecedentes}

La importancia de los indicadores líderes o adelantados es reconocida desde hace varias décadas, en particular porque anticipa la dirección de la actividad económica, proporciona información relevante a los encargados de tomar decisiones de política económica y a los agentes privados. Además, este tipo de indicadores proporcionan información adicional que permite complementar las proyecciones a corto plazo de la producción.

La historia de los indicadores cíclicos inicia a finales de la década de los 30 y principios de los años 40 cuando el National Bureau of Economic Research (NBER) realizó la publicación del trabajo empírico de Burns y Mitchell (1946), donde se proponía una lista de indicadores coincidentes, rezagados y adelantados del ciclo económico de Estados Unidos. La cronología del

\footnotetext{
${ }^{7}$ A diferencia del enfoque cuantitativo, en el que se busca predecir los niveles y/o tasas de crecimiento de una variable agregada, el enfoque cualitativo busca predecir el estado cíclico de la actividad económica.

${ }^{8}$ Otra información relevante se puede revisar en Guidetti y Gyomai (2012).
}

Carlos Chaverri Morales y Diana Van Patten Rivera

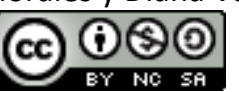

Artículo protegido por licencia Creative Commons 
ciclo económico y la identificación de las series para llevar a cabo la clasificación se basaba en la observación de muchas series de tiempo con datos de diversas actividades.

Dicha entidad hizo publicaciones posteriores que contribuyeron a ampliar los alcances de la técnica propuesta por Burns y Mitchell (1946), como los estudios de Moore y Shiskin (1967), Klein y Moore (1982), y Stock y Watson (1989, 1992).

A partir de los años setenta, la OCDE también creó un sistema para calcular indicadores compuestos. En la actualidad, esta organización realiza la estimación de los indicadores coincidentes, adelantados y rezagados para los países miembros de la organización, el G79, la zona euro y las cinco mayores economías asiáticas.

En lo que respecta al tema de indicadores compuestos en América Latina, los avances son relativamente recientes y se centran en determinar el comportamiento de la actividad económica y la inflación. Entre los países de América del Sur que han realizado varias publicaciones sobre el tema está Chile, donde destacan los trabajos de Marcel y Meller (1983), Crispi (1994) y Bravo y Franken (2002). Posteriormente, Firinguetti y Rubio (2003) estiman un indicador adelantado, en donde utilizan como serie de referencia el índice Mensual de la Actividad Económica (IMACEC).

En el caso de Argentina hay dos estimaciones por considerar, la primera llevada a cabo en el año 2000 por Cerro y Jorrat. Ellos realizaron un cálculo de las probabilidades mensuales asociadas con las variaciones en los puntos de giro tanto en el ciclo económico como el ciclo de crecimiento de la economía argentina, para lo cual emplean la técnica de probabilidades secuenciales recursivas de Neftçi (1982). Los autores construyen además un indicador adelantado y uno coincidente, los cuales se basan en la metodología del NBER. Posteriormente, Melo et al. (2001) estiman un índice coincidente de la actividad económica argentina con un método híbrido, que primero cointegra las series utilizadas y luego aplica la metodología de Stock y Watson $(1989,1992)$.

En Perú, Ochoa y Lladó $(2002,2003)$ realizan dos estudios para estimar indicadores adelantados, en donde se analizaron tanto los puntos de giro como el ciclo de crecimiento de la actividad económica. Por su parte, México realiza estimaciones de indicadores adelantados y coincidentes a la actividad económica de forma mensual.

En Centroamérica y el Caribe, el desarrollo de este tipo de indicadores se ha llevado a cabo por parte de los bancos centrales de Guatemala, Nicaragua, El Salvador, República Dominicana y Costa Rica. En el caso de Guatemala se hace referencia a un indicador adelantado al ciclo de la inflación y se realizó en el año 2000. Por su parte, Fuentes y Salazar (2010) estiman indicador

\footnotetext{
${ }^{9}$ Alemania, Canadá, Francia, Italia, Japón, Reino Unido y Estados Unidos.

60

Carlos Chaverri Morales y Diana Van Patten Rivera

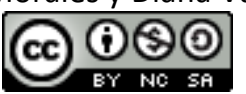

Artículo protegido por licencia Creative Commons
} 
coincidente y otro adelantado a la actividad económica salvadoreña que emplea tanto la metodología del NBER como un modelo factorial. En Costa Rica, en 1993 se estimó un índice compuesto de indicadores monetarios adelantados y posteriormente en 1999 se llevó a cabo la estimación de un indicador adelantado al ciclo inflacionario. Los trabajos a los que se hace referencia son los de Herrera, Ruiz y Valerio (1994), Muñoz y Vindas (1993) y Kikut y Muñoz (1999).

Finalmente, Gallardo y Pedersen (2007) desarrollan un indicador adelantado agregado para América Latina, el cual se valió del método de la OCDE. Ellos parten de un cálculo individual del índice adelantado para once países latinoamericanos y proceden a realizar una agregación.

Para la construcción de los indicadores cíclicos compuestos destacan dos tipos de metodologías: i) el método clásico y ii) los modelos factoriales. El primer método sigue una serie de pasos que incluyen la preselección de variables, el filtrado de las series (desestacionalización), determinación de los puntos de giro, selección de variables y agregación (estos pasos se explican con detalle posteriormente).

Por su parte, el método factorial asume que existen relaciones comunes entre las variables, porque estas son manifestaciones comunes de factores no "observables" de forma directa. Este es similar al método clásico salvo en lo que concierne al filtrado de las series, ya que bajo esta metodología el indicador compuesto se construye a partir de los componentes comunes e idiosincráticos de las series con mayor comunalidad ${ }^{10}$.

\section{Metodología y criterios de selección}

En este documento se sigue la metodología clásica recomendada por la OCDE $(1998,2005)$, procedimientos que han sido complementados con lineamientos dictados por otras instituciones para la selección y tratamiento de las series de tiempo sujetas de estudio.

Al seguir las recomendaciones metodológicas de la OCDE se da un paso importante en lo que concierne al diseño de indicadores cíclicos para la economía costarricense, en particular porque durante el año 2013 el Gobierno de la República presentó una petición formal para que el país sea evaluado para formar parte de la dicha organización.

El primer paso antes de dar inicio a la construcción de los indicadores es la selección de la serie de referencia, seguido por la conformación de una base de datos que cumpla con ciertos requisitos que serán descritos más adelante en esta sección.

\footnotetext{
${ }^{10}$ La comunalidad es la proporción de la varianza explicada por los factores comunes en una variable.
} 


\section{Selección de la serie de referencia}

El ciclo económico (de negocios o tasas de crecimiento) es un fenómeno económico no observable de forma directa, lo cual plantea la necesidad de seleccionar una variable que mida adecuadamente los movimientos de la actividad económica. Este es un paso crucial, porque los indicadores compuestos serán calculados y evaluados en etapas sucesivas, en donde se toma como base la serie de referencia que se elija.

Entre los procedimientos que se emplean para definir la variable de referencia, la literatura cita dos posibilidades. La primera es seguir el método utilizado por la NBER, la OCDE y otras instituciones que consiste en seleccionar una única variable como serie de referencia.

La segunda alternativa sugiere construir un índice de referencia, para ello se utiliza la metodología propuesta por Stock y Watson (1992), quienes proponen medir el "estado de la economía" partiendo de la premisa de que los comovimientos entre series de tiempo que describen distintos sectores de la economía pueden ser capturados por una variable implícita no observable. Lo anterior va de la mano con el supuesto de que la variable no observable es común a las distintas series macroeconómicas y da lugar a un modelo probabilístico donde la serie de referencia es en sí un índice que sintetiza información de otras variables.

En este documento se optó por la primera alternativa descrita y en particular se decidió utilizar como se menciona en la introducción el IMAE con servicios de transformación como la variable para aproximar el "estado de la economía".

\section{Elaboración de la base de datos}

El Business Cycle Indicator Handbook (2001) elaborado por el Departamento del Comercio de Estados Unidos sugiere una lista de recomendaciones que las series deben de cumplir para que sean consideradas en formar parte de la base de datos. Los principios evaluados fueron los siguientes:

i) Importancia económica: la clasificación de los indicadores es un resultado de la sincronización entre la variable y el ciclo económico, pero esta clasificación debe ser racional y coherente con lo que dicta la teoría económica.

ii) Representatividad: las series que finalmente forman parte de cada indicador deben representar a los diferentes sectores que componen la economía.

iii) Ajuste a criterios estadísticos: en esta característica es importante señalar que los datos no solo tienen que ser procesados adecuadamente, deben de cumplir los

62

Carlos Chaverri Morales y Diana Van Patten Rivera

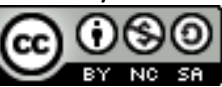

Artículo protegido por licencia Creative Commons 
requisitos estadísticos como el muestreo aleatorio o representatividad de la muestra, sino además deben ser generados por fuentes confiables.

iv) Frecuencia y longitud: para efectos de este trabajo, los datos deben estar disponibles de forma mensual a partir de enero 1996.

v) Series oportunas y no sujetas a revisiones: para continuar la actualización del indicador compuesto mes a mes, los datos deben publicarse de forma oportuna.

vi) Cronología coherente: cada una de las series individuales debe ser clasificada como rezagada, coincidente o adelantada en relación con la serie de referencia.

vii) Suavidad: es preferible que las series que conformen la base de datos no muestren un comportamiento errático o estacional.

viii) Conformidad: un aspecto central es que la serie se adapte bien al ciclo económico, una forma de medir esta conformidad es mediante la correlación entre la serie y la serie de referencia.

\section{Tratamiento y evaluación de las series de tiempo}

Luego de seleccionar las variables que serían considerados en el análisis para obtener los indicadores, cada una de las series fue tratada por aparte con el fin de eliminar el componente estacional, los posibles valores atípicos ("outliers"), tendencia y los componentes irregulares.

\section{Ajustes por estacionalidad}

Para efectuar el ajuste estacional se siguen las recomendaciones del Sistema Estadístico Europeo, el cual sugiere darle un tratamiento individual a cada serie, para cumplir con dicha recomendación se emplearon técnicas de series de tiempo para obtener series desestacionalizadas. El ajuste estacional se hace sobre los niveles de las series. Una vez eliminado el componente estacional las series se transforman en logaritmos.

\section{Extracción del componente cíclico}

El siguiente paso consistió en estimar las series de tendencia para luego estimar el ciclo. Al respecto existen varios métodos recomendados en la literatura empírica con el fin de separar el componente cíclico y la tendencia de una serie de tiempo, en particular, en este documento se seleccionó el filtro Hodrick y Prescott (1980). 
Esta técnica descompone una serie de tiempo en dos componentes, uno de tendencia y otro cíclico. El ajuste de sensibilidad de la tendencia a las fluctuaciones a corto plazo se obtiene con la modificación de un multiplicador $\lambda$ (ecuación 1).

En este proceso, lo que se busca es hacer mínimas las desviaciones de la tendencia, lo cual se captura en el primer componente de la ecuación (1). Lo anterior se lleva a cabo con el objetivo de suavizar la serie para lo cual se impone una penalización por la variación en la segunda diferencia de la tendencia como un criterio de suavidad que se puede observar en el segundo componente de la ecuación (1):

$$
H P=\min _{g_{t}}\left[\sum_{t=1}^{T}\left(y_{t}-g_{t}\right)^{2}+\lambda \sum_{t=1}^{T}\left(\left(g_{t+1}-g_{t}\right)-\left(g_{t-1}-g_{t-2}\right)\right)^{2}\right]
$$

En la ecuación anterior, las desviaciones de la serie con respecto a la tendencia, el ciclo es representado por $c_{t}=y_{t}-g_{t}$. Como se puede apreciar que entre más grande sea el valor de $\lambda$, mayor será la penalización ante variaciones de la tendencia. En el caso de series con periodicidad mensual se suele utilizar $\lambda=14400$. En el caso de Costa Rica, Segura y Vásquez (2011) estimaron un $\lambda=23000$ para series mensuales.

Una vez se estima la serie de tendencia, el ciclo se obtiene como la diferencia entre la serie de tendencia y la serie desestacionalizada.

\section{Análisis del comportamiento cíclico de la serie}

Este paso consistió en un examen gráfico del comportamiento de cada serie, en busca de series que no presentaran un ciclo bien definido. En particular esta etapa de valoración previa permitió identificar series cuyo comportamiento podría afectar la eficacia y la precisión de los indicadores compuestos finales.

\section{Análisis de correlación entre cada serie y la serie de referencia}

Luego del análisis por inspección de los ciclos de las series, y excluidos aquellos considerados como no relevantes, se lleva a cabo un análisis de correlación cruzada y se identifican los puntos de giro de los ciclos de cada una de las variables.

Las correlaciones cruzadas entre cada serie y la serie de referencia se calcularon como una medida de la relación lineal entre las variables, de acuerdo con la siguiente fórmula: 


$$
\rho_{1 \mathrm{i}}(\mathrm{k})=\frac{\operatorname{Cov}\left(\mathrm{z}_{1 \mathrm{t}}, \mathrm{z}_{\mathrm{i}(\mathrm{t}-\mathrm{k})}\right)}{\sqrt{\operatorname{Var}\left(\mathrm{z}_{\mathrm{it}}\right) \operatorname{Var}\left(\mathrm{z}_{1 \mathrm{t}}\right)}}
$$

Para $i=1, \ldots, N$ y $k$. Donde $i$ es el número de variables y $k$ el número de adelantos y rezagos. Con este análisis fue posible obtener tanto las correlaciones cruzadas contemporáneas, así como el período en el que ocurrió la máxima correlación cruzada entre cada variable y la serie de referencia. Lo anterior permite clasificar de forma previa a cada variable: si la máxima correlación cruzada entre cada variable y la serie de referencia se encuentra en $k>2$ la serie se clasifica como que adelantada, si se comprueba para $\mathrm{k}<-2$ esta se clasifica como rezagada, y la serie se clasifica como coincidente si la máxima correlación cruzada entre esta serie y la de referencia se encuentra entre $-2 \leq k \leq 2$.

Además de examinar cuándo ocurre la correlación cruzada máxima, se analiza su magnitud, ya que es deseable incluir series que presentan una correlación con la serie de referencia relativamente fuerte, se decidió aplicar la siguiente regla a los datos como un filtro para que la serie se incluya en la base de datos: si la correlación cruzada máxima $\left(\rho_{\max }\right)$ es inferior a 0,4 en valor absoluto, es decir, $\left|\rho_{\max }\right|<0,4$, entonces la serie se elimina de la base de datos y no se considera en el análisis.

Como resultado de este paso se obtiene una clasificación de las series de datos en adelantadas, rezagadas y coincidentes según la máxima correlación cruzada. Posterior a esto se deben de estudiar los puntos de giro.

\section{Análisis de puntos de giro}

El método utilizado para la detección de puntos de giro sigue el procedimiento de Bry y Boschan (1971). Este método normalmente se inicia con una media móvil para eliminar la tendencia, sin embargo, como en etapas anteriores se filtraron las series, este paso se omite.

Luego, se estima la curva de Spencer mediante la aplicación de una media móvil, del mismo nombre. El inicio de la serie y los puntos finales se extienden bajo el supuesto de que la tasa de crecimiento de las primeras cuatro y de las últimas cuatro observaciones es constante en, respectivamente, los siete períodos anteriores y los siete subsiguientes.

El siguiente paso consiste en detectar valores atípicos y reemplazarlos con sus equivalentes en la curva de Spencer. La media móvil de Spencer se aplica de nuevo, pero en la serie que tiene el valor atípico corregido. Lo anterior da lugar a una curva de Spencer que no presenta los valores atípicos. A continuación, como las series son mensuales, se aplica una media móvil (MA) centrada 2 *12 y se estima una curva para el "primer ciclo". Así, se calcula un "primer conjunto" de puntos de giro y los puntos que corresponden al " primer conjunto " se identifican en la

Carlos Chaverri Morales y Diana Van Patten Rivera

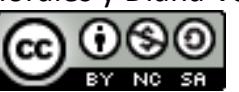

Artículo protegido por licencia Creative Commons 
curva de Spencer. Cabe mencionar que la búsqueda de los puntos de giro se realiza en el rango $[t-5, t+5]$, donde t es un punto que pertenece al llamado "primer conjunto".

La longitud mínima de una fase se encuentra en 1,25*12 períodos (equivalente a 15 meses). La siguiente estimación que es conveniente realizar es la del rezago mensual mínimo para el cual el promedio de las desviaciones absolutas de crecimiento en el ciclo es mayor que el promedio de las desviaciones absolutas de crecimiento en el componente irregular.

Una vez obtenida la longitud de los Meses de Dominancia Cíclica (MDC) ${ }^{11}$, se filtra la serie sin valores atípicos a través de un promedio móvil de precisamente esta longitud. Con esta serie se puede obtener un "segundo conjunto" de puntos de giro que corresponden a los de la curva. En este proceso se impone, nuevamente, una duración mínima de las fases de 1,25*12. Por último, el "tercer conjunto" de puntos de giro se calcula eliminando los puntos situados en las primeros seis y las últimas seis observaciones; esta vez se impone una longitud mínima de fase de cinco observaciones.

Finalmente, se lleva a cabo la comparación entre los puntos de giro de cada serie individual con los que se encuentran en la serie de referencia. Además, las medidas de duración, como la media o la mediana de adelanto o rezago en los picos y valles cíclicos, se calculan con el fin de describir mejor los comovimientos y la relación entre las series.

\section{Construcción del Indicador Compuesto Final}

Los lineamientos de la OCDE para la construcción de indicadores compuestos se basan en el enfoque del ciclo de crecimiento, el cual define un ciclo económico como una desviación de la tendencia de crecimiento a largo plazo, en lugar de definirlo como una disminución o aumento en términos absolutos de la actividad económica agregada.

Bajo el supuesto de que el indicador final estará conformado por $N$ series de tiempo con $n$ observaciones cada una, definidas como $X_{i}$, donde $i=1, \ldots N$, el cálculo del indicador adelantado preliminar se obtiene a través de los siguientes pasos:

\section{Estandarización de $Y_{i}$}

A pesar de que se trabaja con los ciclos de las series clasificadas como adelantadas y en su mayoría estos se derivan de series cuyos niveles son expresados en números índices, es necesario estandarizar. La estandarización de cada serie se logra luego de restarle a cada una su

\footnotetext{
${ }^{11}$ Se refiere al número mínimo de meses que el componente de tendencia ciclo requiere para dominar el componente irregular de una serie de tiempo.
}

66

Carlos Chaverri Morales y Diana Van Patten Rivera

Artículo protegido por licencia Creative Commons 
media, dividirla entre su promedio de desviaciones absolutas y sumarle 100 , de acuerdo con la siguiente fórmula:

$$
S_{t, i}=\frac{C_{t, i}-\bar{C}_{l}}{\left|C_{t, i}-\bar{C}_{l}\right|}+100, \text { para } t=1, \ldots, n
$$

Para $i=1, \ldots N$ y n períodos, donde $\bar{C}_{l}$ representa el valor medio de cada serie.

\section{Agregación de los indicadores}

Con el fin de agregar los componentes del indicador, se calculan las tasas de crecimiento promedio entre dos períodos consecutivos, con la utilización de las series estandarizadas que formarán parte del indicador, condicionadas a que el valor inicial sea igual a $\left(I_{1}=1\right)$; es decir:

$$
I_{t}=\frac{\sum_{i=1}^{N} S_{t, i} \delta_{t-1, i}}{\sum_{i=1}^{N} S_{t-1, i} \delta_{t-1, i}} I_{t-1} \text { para } t=2, \ldots, n
$$

Donde $\delta_{t, i}$ equivale a uno si $S_{t, i}$ está disponible, y a cero en otro caso.

\section{Estandarización del indicador final}

Este es el paso final y consiste en estandarizar el indicador agregado para que fluctúe alrededor de 100. Lo anterior se logra al restarle a $I_{t}$ su media, luego dividir el resultado entre el promedio de desviaciones absolutas de $I_{t}$ y finalmente sumarle 100 , como se describe en la siguiente fórmula:

$$
I C_{t}=\frac{I_{t}-\bar{I}}{\left|I_{t}-\bar{I}\right|}+100 \text { para } t=1, \ldots, n
$$

\section{Datos utilizados}

En esta sección se lleva a cabo una descripción de los datos utilizados. En el análisis, la información que se toma en cuenta se ve definida por dos aspectos relevantes los cuales han sido mencionados con antelación: i) la disponibilidad para un período suficiente, y ii) el hecho de que todas las series seleccionadas deben tener significancia económica.

El trabajo se desarrolla sobre la base de cifras mensuales con el fin de aprovechar la producción estadística que lleva a cabo el Departamento de Estadística Macroeconómica del Banco Central de Costa Rica. Además, el análisis busca que las series candidatas a formar parte del indicador compuesto representen distintos sectores de la economía. En línea con lo anterior, se cuenta con indicadores monetarios, del mercado laboral, del sector real (principalmente la información que se utiliza para la construcción del IMAE), variables del sector externo (precios internacionales, actividad económica de los socios comerciales y datos del comercio exterior),

Carlos Chaverri Morales y Diana Van Patten Rivera

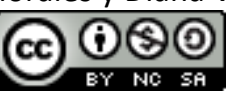

Artículo protegido por licencia Creative Commons 
índices de precios (al consumidor, de los servicios y los precios al productor industrial) y un grupo de otras variables relevantes.

\section{Tabla 1.}

Agrupación y número de datos para la construcción de los indicadores compuestos ${ }^{12}$

\begin{tabular}{llc}
\hline \multicolumn{1}{c}{ Grupo } & Cantidad de variables \\
\hline 1. & Variables monetarias & 9 \\
2. Variables del sector real & 199 \\
3. Mercado laboral & 2 \\
4. Índices de precios & 15 \\
5. Sector externo & 36 \\
6. Sector fiscal & 3 \\
7. & Otros indicadores & 5 \\
\hline
\end{tabular}

Fuente: elaboración propia.

Otro aspecto importante tiene que ver con el nivel de detalle de la información, principalmente con las actividades del sector real cuyo nivel de agregación puede llegar a proporcionar información por producto. Tres niveles de agregación se utilizan, que de forma general incluyen la información de los grupos 1 al 6 con combinaciones de información detallada del IMAE por subgrupos. Así por ejemplo, el nivel de agregación 1 (en adelante nivag1) combina datos de los agregados monetarios, mercado laboral, índices de precios y algunos indicadores del sector externo con datos de las industrias que componen el IMAE por actividad productiva. Adicionalmente, en la construcción del indicador se usan dos niveles de agregación adicionales: nivel de agregación 2 (nivag2) y el nivel de agregación 3 (nivag3). El nivel de agregación 2 contiene información desagregada de las industrias que componen el IMAE y el nivag3 incorpora información más detallada, en algunos casos a nivel de producto y en otros casos indicadores de cantidad como lo es el caso del turismo, actividad sobre la cual se dispone el número de turistas que ingresan al país de forma mensual.

En total se dispone de una base de 270 series con periodicidad mensual. Con el fin de usar la mayoría de información disponible y garantizar la evaluación de información de distintos sectores se decide iniciar el análisis a partir de enero de 1996. Con esto la base se reduce a 90 variables, cada una con información completa para el período de estudio.

La construcción de un indicador compuesto requiere que la información base utilizada sea en términos reales, por lo que los datos del sector monetario se emplean en términos reales deflactados con el IPC.

\footnotetext{
${ }^{12}$ No se dispone de una desagregación de las exportaciones e importaciones por producto en términos reales.
} 68

Carlos Chaverri Morales y Diana Van Patten Rivera

Artículo protegido por licencia Creative Commons 


\section{Resultados obtenidos}

Como se citó en secciones previas, la variable de referencia seleccionada fue el IMAE, el ciclo de esta variable se obtuvo mediante la estimación de las desviaciones cíclicas con respecto a la trayectoria a largo plazo.

Como primer resultado se obtiene la siguiente cronología del ciclo de crecimiento del IMAE ${ }^{13}$.

\section{Tabla 2.}

Cronología del ciclo del IMAE

\begin{tabular}{ccc}
\hline & Tipo & Fecha \\
\hline 1 & Valle & Ago. 1997 \\
2 & Pico & Mar. 1999 \\
3 & Valle & Set. 2001 \\
4 & Pico & Feb. 2003 \\
5 & Valle & Set. 2004 \\
6 & Pico & Jun. 2007 \\
7 & Valle & Ene. 2009 \\
8 & Pico & Abr. 2010 \\
9 & Valle & Nov. 2010 \\
10 & Pico & Mar. 2012 \\
11 & Valle & Oct. 2012 \\
\hline
\end{tabular}

Fuente: elaboración propia.

La estimación de las distintas versiones de los indicadores se llevó en tres etapas para facilitar su comparación. En la primera etapa se consideraron todas las variables clasificadas como nivel de agregación 1 y se impusieron a priori dos criterios de selección: el primero que las variables seleccionadas solamente fueran adelantadas respecto a los movimientos cíclicos de la variable de referencia y en segundo lugar que el coeficiente de correlación de los ciclos entre las variables fuera superior a 0,4.

Este primer ejercicio permitió identificar las siguientes variables como buenas candidatas para proporcionar información adelantada en relación con los puntos de giro de la actividad económica: índice mensual de Agricultura, silvicultura y pesca, empleo sin cuenta propia, tipo de cambio nominal, IMAE de El Salvador, indicadores compuestos líderes de Estados Unidos y la Eurozona, ingresos tributarios en términos reales y los índices de precios de los siguientes grupos del IPC: comidas y bebidas fuera del hogar, prendas de vestir y calzado, artículos para la vivienda y servicio doméstico, entretenimiento y cultura. Una vez normalizadas y agregadas de forma ponderada en un solo indicador. Su comportamiento se describe en la figura 1.

${ }^{13}$ Estos resultados pueden variar con respecto a los obtenidos en Chaverri (2011) por el método utilizado. 


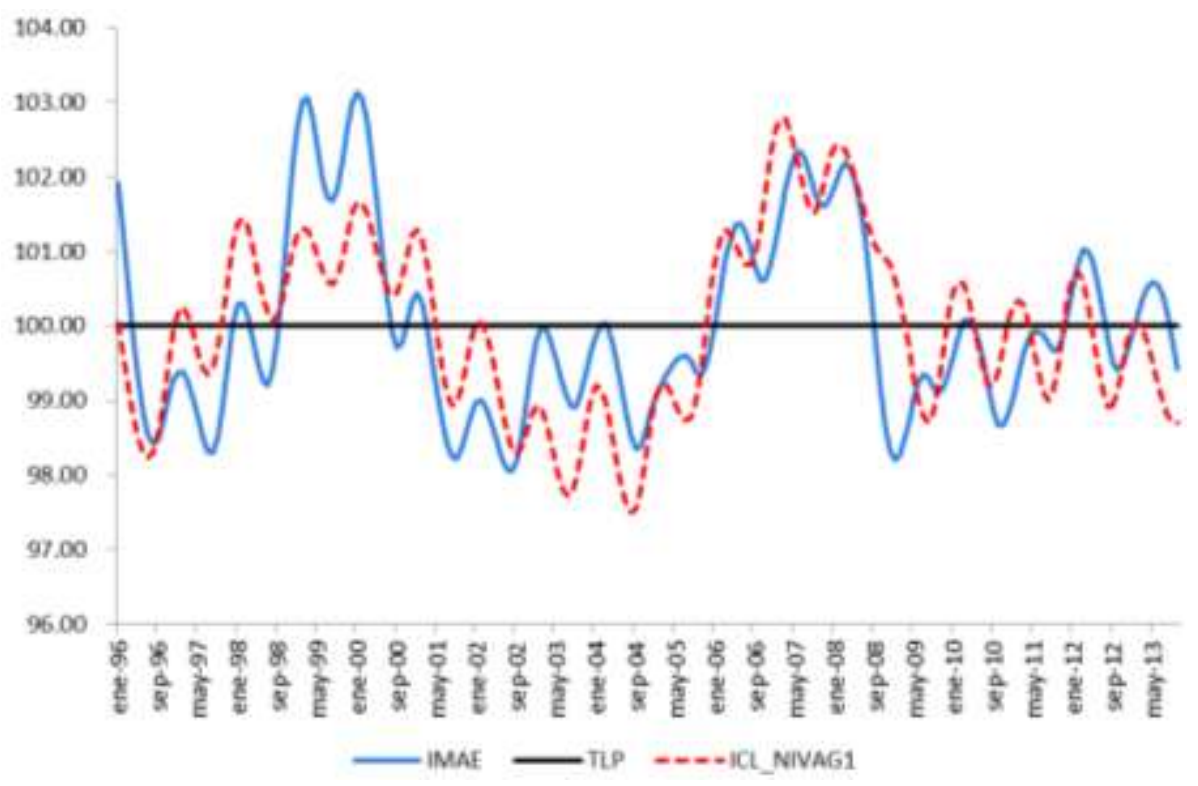

Figura 1. Ciclo del IMAE e indicador compuesto adelantado $1^{14}$. Fuente: elaboración propia.

Los valores por encima de la línea de 100 y que se incrementan indican que la actividad económica se encuentra en una fase de expansión, mientras valores por encima de 100, pero que disminuyen sugieren que la economía se encuentra en una fase de desaceleración. Adicionalmente, valores menores a 100 y que disminuyen son indicativos de una contracción y valores menores a 100 pero que se incrementan son indicativos de una recuperación.

Este primer indicador registró un adelanto medio de 13 meses y una correlación con respecto al ciclo del IMAE de 0,73. Cuando se agrega la información, el indicador compuesto únicamente registra un punto de giro perdido con respecto a los que se encuentran en la variable de referencia y cuatro puntos extra en el indicador que no están originalmente en la serie del ciclo del IMAE.

\footnotetext{
${ }^{14}$ La línea negra horizontal corresponde a la tendencia a largo plazo que se define sobre el valor de 100. 70

Carlos Chaverri Morales y Diana Van Patten Rivera

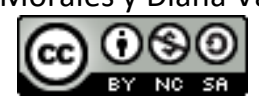

Artículo protegido por licencia Creative Commons
} 
Tabla 3.

Cronología del indicador compuesto adelantado 1

\begin{tabular}{ccrl}
\hline Puntos de giro & Tipo & Fecha & \multicolumn{1}{c}{ Relación con ciclo del IMAE } \\
\hline 1 & Valle & Jul. 1996 & Adelanta 13 meses ago. 1997 \\
2 & Valle & Ago. 1998 & Extra \\
3 & Pico & Feb. 1998 & Adelanta 13 meses mar. 1999 \\
4 & Pico & Ene. 2000 & Extra \\
5 & Valle & Ago. 2001 & Adelanta 1 mes set. 2001 \\
6 & Pico & Ene. 2002 & Adelanta 13 meses feb. 2003 \\
7 & Valle & Jul. 2003 & Adelanta 14 meses set. 2004 \\
8 & Valle & Ago. 2006 & Extra \\
9 & Pico & Mar. 2006 & Adelanta 15 meses jun. 2007 \\
10 & Pico & Feb. 2008 & Extra \\
11 & Valle & Ene. 2009 & Perdido \\
12 & Pico & Feb. 2010 & Adelanta 2 meses abr. 2001 \\
13 & Valle & Ago. 2009 & Adelanta 15 meses nov. 2010 \\
14 & Pico & Feb. 2012 & Adelanta 1 mes mar. 2012 \\
15 & Valle & Ago. 2011 & Adelanta 14 meses oct. 2012 \\
\hline
\end{tabular}

Fuente: elaboración propia.

El segundo ejercicio consistió en utilizar la información con un nivel de agregación 2, es decir, se incluía información con un nivel de detalle según la clasificación industrial internacional uniforme CIIU a dos dígitos. Con esto se evita usar los índices globales de las industrias y se obtiene una mejora al emplear información más puntual que en el caso previo.

Como resultado de esta modificación, en la información base se puede identificar que el índice mensual de Exportables, Otros Agropecuarios, el indicador de Manufactura del régimen definitivo, el índice de Muelles y Puertos, el índice de Autos de Alquiler, Empleo sin Cuenta propia, la tasa Libor a 6 meses, el Indicador Compuesto líder de EUA y de la Eurozona, los ingresos tributarios en términos reales y los índices de precios de los siguientes grupos del IPC: i)comidas y bebidas fuera del hogar, ii) prendas de vestir y calzado, iii) artículos para la vivienda y servicio doméstico, y iv) entretenimiento y cultura conforman el grupo de variables que brinda información oportuna acerca de los cambios en la actividad económica. Como se puede apreciar algunas de estas series coinciden con las del caso previo. 


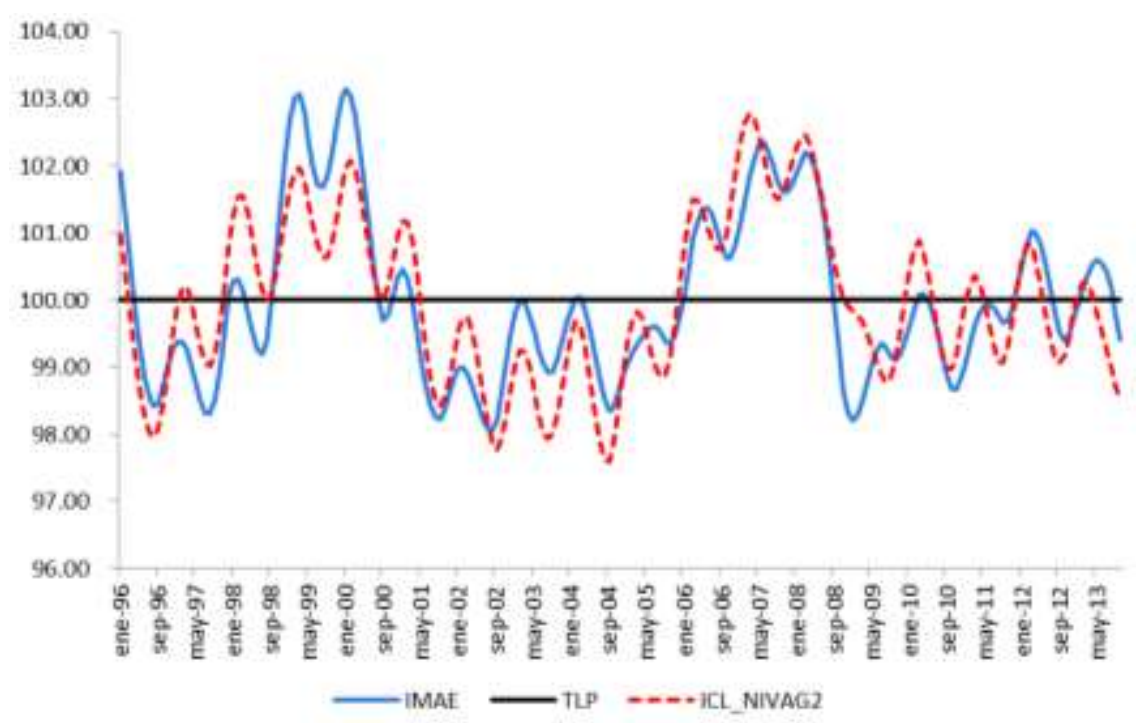

Figura 2. Ciclo del IMAE e indicador compuesto adelantado 2.

Fuente: elaboración propia.

Este segundo indicador registró una mediana de los adelantos de 12 meses y una correlación con respecto al ciclo del IMAE de 0,83. El análisis de la cronología con respecto al ciclo de referencia permite identificar un punto de giro perdido; cuatro giros adicionales y dos que coinciden con dos puntos de giro del IMAE.

\section{Tabla 4.}

Cronología del indicador compuesto adelantado 2

\begin{tabular}{cccl}
\hline Puntos de giro & Tipo & Fecha & \multicolumn{1}{c}{ Relación con ciclo del IMAE } \\
\hline 1 & Valle & 1996.8 & Adelanta 12 meses ago. 1997 \\
2 & Valle & 1998.8 & Extra \\
3 & Pico & 1998.3 & Adelanta 12 meses mar. 1999 \\
4 & Pico & 2000.2 & Extra \\
5 & Valle & 2001.9 & Coincide con set. 2001 \\
6 & Pico & 2002.2 & Adelanta 12 meses feb. 2003 \\
7 & Valle & 2003.8 & Adelanta 13 meses set. 2004 \\
8 & Pico & 2005.3 & Extra \\
9 & Valle & 2005.8 & Extra \\
10 & Pico & 2007.3 & Adelanta 3 meses jun. 2007 \\
11 & Valle & 2009.1 & Perdido \\
12 & Pico & 2010.3 & Adelanta 1 mes abr. 2010 \\
13 & Valle & 2009.8 & Adelanta 15 meses nov. 2010 \\
14 & Pico & 2012.3 & Coincide con mar. 2010 \\
15 & Valle & 2011.9 & Adelanta 13 meses oct. 2012 \\
\hline
\end{tabular}

Fuente: elaboración propia.

72

Carlos Chaverri Morales y Diana Van Patten Rivera

(c) (1) (1) (2)

Artículo protegido por licencia Creative Commons 
Finalmente, el tercer ejercicio consistió en utilizar un nivel de detalle más profundo en la información básica, principalmente de la industria manufacturera. Esta vez en lugar de usar datos a nivel de CIIU a dos dígitos se emplea información a nivel de productos según la Clasificación Central de Productos (CCP).

Al igual que en los ejercicios previos, algunas series se repiten dentro de las elegidas para formar parte del indicador final. Tal es el caso de indicadores compuestos líderes de Estados Unidos y la Eurozona, los ingresos tributarios en términos reales y los índices de precios del IPC: comidas y bebidas fuera del hogar, prendas de vestir y calzado, artículos para la vivienda y servicio doméstico, entretenimiento y cultura y otras ingresan, como por ejemplo: producción de cacao, producción de piña, producción de leche, electricidad industrial menor (medida en $\mathrm{MWH}$ ), total de turistas que ingresan al país, el de total de toneladas movilizadas por los puertos (exportaciones e importaciones) y las exportaciones movilizadas por todos los muelles y puertos.

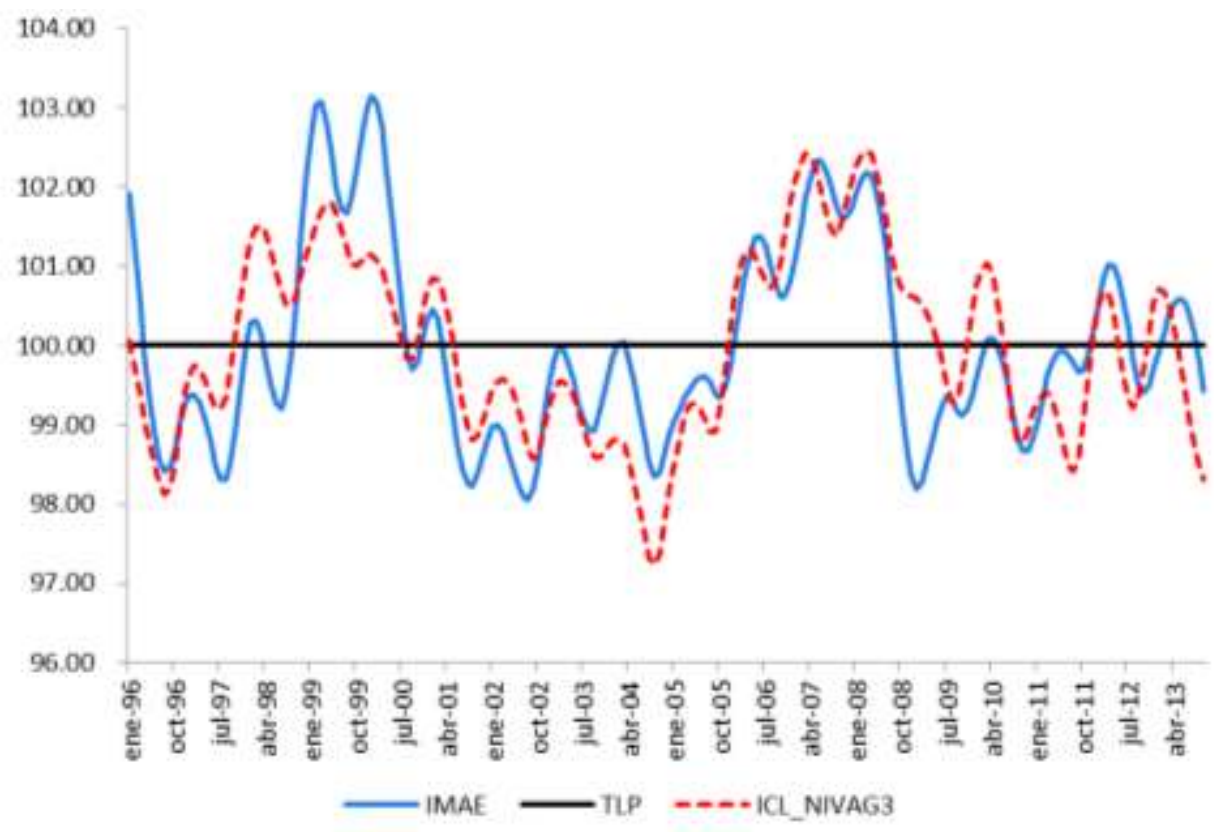

Figura 3. Ciclo del IMAE e indicador compuesto adelantado 3.

Fuente: elaboración propia

No obstante, en este caso del indicador compuesto adelantado, la mediana del adelanto es de 12 meses con una correlación de 0,75. Esta tercera variante en la construcción del indicador compuesto sugiere preliminarmente un pico en la actividad económica durante enero de 2013. 


\section{Tabla 5.}

Cronología del indicador compuesto adelanto 3.

\begin{tabular}{cccl}
\hline Puntos de giro & Tipo & Fecha & \multicolumn{1}{c}{ Relación con ciclo del IMAE } \\
\hline 1 & Valle & Ago. 1996 & Adelanta 12 meses ago. 1997 \\
2 & Pico & Mar. 1998 & Adelanta 12 meses mar. 1999 \\
3 & Pico & Ene. 2001 & Extra \\
4 & Valle & Ago. 2000 & Adelanta 13 meses set. 2001 \\
5 & Valle & Set. 2002 & Extra \\
6 & Pico & Mar. 2003 & Rezagado 1 mes feb.2003 \\
7 & Valle & Set. 2004 & Coincide con set. 2004 \\
8 & Pico & Mar. 2007 & Adelanta 3 meses jun. 2007 \\
9 & Valle & Ene. 2009 & Perdido \\
10 & Pico & Mar. 2010 & Adelanta 1 mes abr. 2010 \\
11 & Valle & Ago. 2009 & Adelanta 15 meses nov. 20101 \\
12 & Pico & Mar. 2012 & Perdido \\
13 & Valle & Ago. 2011 & Adelanta 14 meses oct. 2012 \\
14 & Pico & Ene. 2013 & Preliminar \\
\hline
\end{tabular}

Fuente: elaboración propia

\section{Conclusiones}

En este documento de investigación se describió el proceso de elaboración de una serie de índices compuestos adelantados con periodicidad mensual con el fin de identificar posibles puntos de giro de la actividad económica costarricense. Para ello se han elaborado tres versiones del indicador, en donde se tiene como diferencia el tipo de información y el nivel de detalle utilizado en cada uno.

Inicialmente se analizaron 270 variables, las cuales contienen información del sector monetario, real, mercado laboral, índices de precios, indicadores del sector externo y variables del sector fiscal. Una vez que la muestra se ajusta para tener series con información completa se dispone de una base de datos de 90 variables con información a partir de enero 1996.

La información del sector real se desagrega en tres niveles, en el cual se pasa de una clasificación por industrias a dos dígitos, según la clasificación CIIU, a una desagregación a nivel de productos para el sector agropecuario e industrial.

Con lo anterior se logró identificar una serie de variables que aportan información adelantada con respecto a los puntos de giro de la actividad económica. Tal es el caso del empleo sin cuenta propia (6 meses), tasa Libor a 6 meses (7,9 meses), indicador compuesto líder de EUA (5,7 
meses), indicador compuesto líder de la Eurozona (7,1 meses), IPC comidas y bebidas alcohólicas (8,3 meses), IPC prendas de vestir y calzado (6,3 meses), IPC artículos para la vivienda y servicio doméstico (6,4 meses), IPC entretenimiento y cultura $(10,6$ meses) y los ingresos tributarios en términos reales (5,0 meses).

Al combinar las variables anteriores se construyeron tres indicadores adelantados con variables cuyo ciclo tuvieran una correlación en valor absoluto mayor a 0,4 con respecto al ciclo de la variable de referencia. El adelanto promedio de los indicadores líderes es de 10 meses para el caso del nivel de agregación 1; 8,1 meses para el nivel de agregación 2, mientras el indicador del nivel de agregación 3 tiene un adelanto promedio de 7,7 meses.

El estudio recomienda la construcción y seguimiento del indicador compuesto adelantado 2 por dos motivos: i) es el indicador cuya correlación con el IMAE es mayor, y ii) evita el uso de indicadores ponderados que ya son parte del IMAE como en el caso del nivel de agregación 1 o muy detallados como el caso del nivel de agregación 3.

Para finalizar, es importante recalcar que conforme la economía va experimentando transformaciones en su sistema productivo, es posible que la relación cíclica entre algunos indicadores cambie. Por lo tanto, la revisión de los indicadores y sus componentes es un trabajo continuo y debe realizarse periódicamente.

\section{Referencias}

Abad, A., Cristóbal, A. y Quilis, E. (1994). El Análisis de la Coyuntura en el Instituto Nacional de Estadística. Informe para el proyecto Tools and Practices For Business Cycle Analysis in National Statistical Institutes of EU (BUSY). WP4: Methods for official statistics - The practicioner side. Recuperado de http://www.ine.es/daco/daco42/daco4214/cbtc17.pdf

Bravo, H. y Franken, H. (Junio, 2002). Un indicador líder del IMACEC. Revista de Análisis Económico, 17(1), 103-123. Recuperado de http://www.raeear.org/index.php/rae/article/view/16

Bry, G. y Boschan, Ch. (1971). Standard Business Cycle Analysis Of Economic Time Series. En G. Bry y Ch. Boschan (Eds.), Cyclical Analysis of Economic Time Series: Selected Procedures and Computer Programs (64-150). Estados Unidos: National Bureau of Economic Research (NBER), Inc. Recuperado de http://www.nber.org/chapters/c2149.pdf

Burns, A. y Mitchell, W. (1946). Measuring Business Cycles. New York: The National Bureau of Economic Research (424-471). Recuperado de http://www.nber.org/chapters/c2990.pdf

Carlos Chaverri Morales y Diana Van Patten Rivera

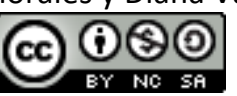

Artículo protegido por licencia Creative Commons 
Cerro, A. y Jorrat, J. (2000). Computing turning point monthly probability of the Argentinian economy according to the leading index: 1973-2000. Estudios de Economía, 27(2), 279295. Recuperado de http://www.estudiosdeeconomia.cl/publicacion/show/id/292

Chaverri, C. (2011). Cronología del ciclo económico de Costa Rica 1976-2010. Manuscrito inédito, Departamento de Investigación Económica, Banco Central de Costa Rica, Costa Rica. Recuperado de http://www.bccr.fi.cr/investigacioneseconomicas/crecimientoeconomico/Cronologia Ci clo Economico en Costa Rica 1976-2010.pdf

Chaverri, C. y Rodríguez, A. (2013). Hechos estilizados de la economía costarricense. Manuscrito inédito, Departamento de Investigación Económica, Banco Central de Costa Rica, Costa Rica. Recuperado de http://www.bccr.fi.cr/investigacioneseconomicas/crecimientoeconomico/Hechosestiliza doseconomacostarricense91 2012.pdf

Crispi, J. (1994). Indicadores líderes en Chile: diseño y extracción de señales. Chile: Instituto Nacional de Estadística.

Firinguetti, y Rubio (Abril, 2003). Indicadores líderes del IMACEC. Manuscrito inédito, Banco Central de Chile, Chile. Recuperado de http://www.bcentral.cl/estudios/documentostrabajo/pdf/dtbc208.pdf

Fuentes, J. y Salazar, R. (julio-setiembre, 2010). Cálculo de un indicador coincidente y adelantado de la actividad económica salvadoreña. Monetaria, 33(3), 289-376. Recuperado de http://www.cemla.org/PDF/monetaria/PUB MON XXXIII-03.pdf

Gallardo, M. y Pedersen, M. (mayo, 2007). Un sistema de indicadores líderes compuestos para la región de América Latina. Estudios Estadísticos y Prospectivos Series (51), 7-63. Recuperado http://repositorio.cepal.org/bitstream/handle/11362/4758/S0700434 es.pdf?sequence $=1$

Guidetti, E. y Gyomai, G. (Abril, 2012). OECD System of composite leading indicators. Recuperado de http://www.oecd.org/std/leading-indicators/41629509.pdf

Herrera, R. y Valerio, D. (1994). Índice de quantum para las importaciones de Costa Rica y su aplicación en la construcción de un indicador adelantado de la actividad económica 1979-1992 (Tesis de Licenciatura inédita). Universidad de Costa Rica, Costa Rica. 
Hodrick y Prescott (1980). Postwar U.S. Business Cycles: An Empirical Investigation. Journal of money, credit, \& banking 29(1), 1-16. Recuperado de https://imcb.osu.edu/archive/volume-29

INEGI. (2010). Sistema de indicadores compuestos coincidente y adelantado. México: INEGI. Recuperado http://www3.inegi.org.mx/sistemas/biblioteca/ficha.aspx?upc=702825000346

Kikut, A. y Muñoz, E. (1999). Diseño de un índice sintético adelantado para la inflación: el caso de Costa Rica. Recuperado de http://www.bccr.fi.cr/investigacioneseconomicas/politicamonetariaeinflacion/Diseno in dice sintetico adelantado para la inflacion.pdf

Klein, P. y Moore, G. (1982). The Leading Indicator Approach To Economic Forecasting Retrospect and Prospect. (Working Papers 941). Recuperado de National Bureau of Economic Research: http://www.nber.org/papers/w0941.pdf

Marcel, M. y Meller, P. (1983). Indicadores Líderes de Recesión y Expansión Económica (Estudio № 75). Recuperado del sitio de internet de la Corporación de Estudios para Latinoamérica: http://www.cieplan.org/media/publicaciones/archivos/121/Capitulo 3.pdf

Melo, L., Nieto, F., Posada, C., Betancourt, Y. y Barón, J. (2001). Un índice coincidente para la actividad económica colombiana (Borradores Semanales de Economía 195). Recuperado de http://www.banrep.gov.co/docum/ftp/borra195.pdf

Moore, G. y Shiskin, J. (1967). Indicators of Business Expansions and Contractions. Estados Unidos: National Bureau of Economic Research. Recuperado de http://papers.nber.org/books/moor67-2

Muñoz, E. y Vindas, K. (1993). Construcción de un índice compuesto de indicadores monetarios adelantados como técnica de pronóstico: caso de Costa Rica 1976-1991. Costa Rica: Banco Central de Costa Rica. Recuperado de http://books.google.co.cr/books/about/Construcci\%C3\%B3n de un indice compuesto de.html?id=BzZTGwAACAAJ\&redir esc=y

Neftçi, S. (1982). Optimal Prediction of Cyclical Downturns. Journal of Economic Dynamics and Control, 4, 225-241. doi:10.1016/0165-1889(82)90014-8 
Ochoa, E. y Lladó, J. (2003). Modelos de indicadores líderes de actividad económica para el Perú. Revista Estudios Económicos, (issue 10). Recuperado de http://econpapers.repec.org/article/rbpesteco/ree-10-04.htm

Ochoa, E. y Lladó, J. (Enero, 2002). Proyección de la actividad económica para la economía Peruana: el uso de indicadores líderes. Trabajado presentado en el XVIII Encuentro de Economistas del Banco Central de Reserva del Perú, Perú.

OECD (1998). OECD Composite Leading Indicators: a tool for short-term analysis. Recuperado de http://www.oecd.org/std/leading-indicators/15994428.pdf

OECD (2005). OECD Cyclical Analysis and Composite Indicators System. User Guide. Recuperado de http://www.cepal.org/deype/noticias/noticias/1/20961/oecd CACIS UserGuidePatV3.pdf

Segura, C. y Vásquez, J. (2011). Estimación del parámetro de Suavizamiento del filtro de Hodrick y Prescott para Costa Rica. Manuscrito inédito, recuperado del sitio del Departamento de Investigación Económica, Banco Central de Costa Rica: http://www.bccr.fi.cr/investigacioneseconomicas/metodoscuantitativos/Estimacion del parametro de suavizamiento del filtro de Hodrick y Prescott para CR.pdf

Stock, J. y Watson, M. (1988). A Probability Model of the Coincident Economic Indicator (Working Paper \#2772). Recuperado de The National Bureau of Economic Research: http://www.nber.org/papers/w2772

Stock, J. y Watson, M. (1989). New Indexes of Coincident and Leading Economic Indicators.. Recuperado de http://papers.nber.org/books/blan89-1

Stock, J. H. y Watson, M. (1992). A procedure for predicting recessions with leading indicators: econometric issues and recent performance (Working Papper No.4014). Recuperado de The National Bureau of Economic Research: http://www.nber.org/papers/w4014.pdf

The Conference Board (2001). Business Cycle Indicators Handbook. Recuperado de http://www.conference-board.org/pdf free/economics/bci/BCl-Handbook.pdf

Zarnowitz, V. (1992). Business Cycles: Theory, History, Indicators, and Forecasting. Estados Unidos: University of Chicago Press. Recuperado de: http://papers.nber.org/books/zarn92-1 